\title{
Utilidad de la consulta no presencial en un Servicio de Cirugía durante la pandemia COVID
}

\author{
Utility of the non-face-to-face consultation in a Surgery \\ Service during the COVID pandemic
}

\author{
Enrique Calcerrada Alises, ${ }^{*}$ Armando Galván Pérez, ${ }^{*}$ Ana Minaya Bravo, ${ }^{*}$ Carlos San Miguel Méndez, ${ }^{*}$ \\ Marina Pérez-Flecha González, ${ }^{*}$ Joaquín Manuel Muñoz Rodríguez, ${ }^{*}$ Jaime Ruíz Tovar, ${ }^{*}$ Almudena Moreno Elola-Olaso, \\ Patricia López Quindós, ${ }^{*}$ Enrique González González, ${ }^{*}$ Arturo Cruz Cidoncha, ${ }^{*}$ Carmen Jiménez Ceinós, ${ }^{*}$ \\ Asunción Aguilera Velardo, ${ }^{*}$ Álvaro Robin Valle-De Lersundi, ${ }^{*}$ Juan Gabriel Tejerina, ${ }^{*}$ Miguel Ángel García Ureña* \\ * Servicio de Cirugía General y del Aparato Digestivo. Hospital Universitario del Henares. Madrid, España.
}

RESUMEN

Introducción: La enfermedad por coronavirus (COVID-19) se ha propagado de forma rápida alrededor del mundo produciendo una pandemia. Con el objetivo de frenar su avance, se ha modificado la estructura asistencial de todos los hospitales, priorizando a los pacientes COVID y urgentes, y paralizando todas las actividades que puedan ser aplazadas. Uno de los principales elementos afectados han sido las consultas, habitualmente presenciales, dado que el objetivo es reducir la exposición de pacientes y personal médico al virus. Como el seguimiento de nuestros pacientes no puede abandonarse, la teleconsulta se presenta como una alternativa eficaz durante la pandemia. Material y métodos: En nuestro centro hemos optado por la consulta telefónica durante los meses de máxima incidencia de la pandemia (marzo-mayo). Esto nos ha permitido realizar el seguimiento de 2,120 pacientes que estaban citados en esas fechas. Resultados: Tras una primera llamada, dividimos a los pacientes en varios grupos según su prioridad para las consultas sucesivas: urgente $(0.09 \%)$, preferente $(10.61 \%)$ y sin prioridad $(60.75 \%)$. Aquéllos que estaban asintomáticos fueron dados de alta (19.05\%). Conclusiones: La consulta telefónica se ha mostrado como una forma eficaz de mantener el seguimiento de nuestros casos durante la pandemia. Más allá de este periodo, en el futuro, podría mostrarse como una alternativa eficaz para el seguimiento de ciertos pacientes y patologías seleccionados.

Palabras clave: Telemedicina, consulta telefónica, teleconsulta.

\section{ABSTRACT}

Introduction: Coronavirus disease (COVID-19) has spread rapidly around the world causing a pandemic. With the aim of slowing down its progress, the healthcare structure of all hospitals has been modified, prioritizing COVID and urgent patients and paralyzing all those activities that could be delayed. One of the main elements affected has been consultations, usually face-to-face, since the objective is to reduce the exposure of patients and medical personnel to the virus. Since the followup of our patients cannot be abandoned, teleconsultation appears as an effective alternative during the pandemic. Material and methods: In our center we have opted for telephone consultation during the months with the maximum incidence of the pandemic (March-May). This has allowed us to monitor 2120 patients who were cited on those dates. Results: After a first call, we divided the patients into several groups according to their priority for subsequent consultations: urgent $(0.09 \%)$, preferential (10.61\%) and no priority (60.75\%). Those who were asymptomatic were discharged (19.05\%). Conclusions: Therefore, telephone consultation has been shown to be an effective way of keeping track of our patients during the pandemic. Beyond this period, in the future, it could be shown as an effective alternative for the monitoring of certain selected patients and pathologies.

Keywords: Telemedicine, telephone consultation, teleconsultation. 


\section{INTRODUCCIÓN}

La enfermedad por coronavirus (COVID-19) fue identificada inicialmente en Wuhan (China). En cuestión de meses se propagó por todo el mundo, por lo que la Organización Mundial de la Salud (OMS) la catalogó como pandemia el 11 de marzo de 2020. Su rápida transmisión y alta tasa de hospitalizaciones ha obligado a paralizar la práctica clínica habitual para conseguir frenar su avance. La situación del personal sanitario en primera línea de combate lo ha convertido en uno de los grupos más vulnerables. ${ }^{1}$

Dada la importancia del caso, numerosas organizaciones mundiales han recomendado cesar todas las actividades hospitalarias no preferentes para reducir la exposición de los pacientes al virus..$^{2-4}$

Los hospitales de los países afectados se han visto obligados a modificar su estructura asistencial, dando prioridad a los pacientes COVID y patologías urgentes, y posponiendo todas las intervenciones quirúrgicas, pruebas y consultas que puedan ser aplazadas.

Sin embargo, es evidente que el seguimiento de nuestros casos no puede abandonarse, lo que ha obligado a buscar nuevas formas de mantener el contacto con ellos. Es aquí donde la tecnología juega un papel fundamental.

Este hecho ha condicionado la actividad de nuestra especialidad, entre otros aspectos, en la distribución de las consultas de Cirugía General, antes realizadas de manera presencial, y nos ha obligado a utilizar nuevas formas de comunicación a distancia que permitan el seguimiento y reduzcan el riesgo de contagio de pacientes y personal sanitario.

El objetivo de este estudio es presentar los resultados de la propuesta de entrevista telefónica para sustituir la consulta presencial, y poder agilizar y priorizar la lista de espera de consulta habitual cuando la situación de la pandemia lo permita.

\section{MATERIAL Y MÉTODOS}

La capacidad máxima de nuestro hospital es de 250 camas y atiende una población de 170,000 habitantes en la periferia de Madrid. El servicio cuenta con una plantilla de 12 cirujanos, por lo general, se dispone de 11 sesiones de quirófanos semanales y 12 consultas semanales. Dispone de historia clínica electrónica desde el comienzo de la creación del hospital en el año 2008 con el software «Selene», la cual permite la localización y acceso ágil a la historia clínica de los pacientes.

El Servicio de Cirugía General y Aparato Digestivo atiende la consulta genérica propia de la especialidad y, además, tiene consultas monográficas de mama, coloproctología, pared abdominal y de pacientes oncológicos nuevos. Cada mes, se atienden alrededor de 1,248 pacientes en consultas.
La interrupción brusca de toda actividad asistencial en el momento de la pandemia (semana del 10 de marzo) obligó a suspender todas las consultas (pacientes nuevos, revisiones posoperatorias y pruebas diagnósticas). Sólo se mantuvo una consulta semanal de pacientes oncológicos. Desde el inicio de la pandemia, los cirujanos fuimos asignados a trabajar en diferentes tareas siguiendo las instrucciones desde el gabinete de crisis, creado para poder combatir los efectos de la pandemia, por lo que se abandonó la cirugía electiva y las consultas habituales. Los cirujanos hemos participado de manera activa colaborando con el Servicio de Urgencias, Medicina Interna y Cuidados Intensivos. Cuando se comenzaron a reducir estas necesidades, se planteó la posibilidad de recuperar las consultas canceladas y establecer un circuito de priorización de las consultas presenciales cuando se restauren.

Se solicitó al Departamento de Informática que proporcionara los listados en Microsoft Excel de los sujetos cancelados y pendientes de cita.

En nuestro caso, hemos optado por la consulta telefónica, se llamó a cada persona que aparecía en el listado para conocer la situación de todos. Según el caso, algunos pacientes fueron dados de alta por mejoría posoperatoria o por no requerir más pruebas y seguimiento. El resto se distribuyó según prioridad en tres grupos: urgentes, los que requerían una revisión presencial en menos de una semana por encontrarse muy sintomáticos y requerir una cirugía o prueba prioritarias en menos de una semana; preferentes, los que deben valorarse en menos de un mes ya sea para realizar una prueba o programar una intervención quirúrgica; y no prioritarios, los que pueden demorarse entre tres y seis meses. Ejemplos de ello se muestran en la Tabla 1.

Para sujetos en seguimiento crónico, les consultamos su situación, revisamos las pruebas realizadas y, en caso de no haber incidencias, se solicitaron las siguientes pruebas de control y cita sucesiva.

\section{RESULTADOS}

Al inicio de la pandemia, en marzo de 2020, estaban citados 3,750 pacientes hasta marzo de 2021. Durante el periodo marzo-mayo llamamos a un total de 2,120 personas para conocer su situación actual. Tras esta primera consulta, se dividieron según prioridad para las sucesivas (Figura 1). En estos momentos, la previsión es aumentar de forma gradual las consultas presenciales mientras se compaginan con las telefónicas, a medida que se progresa en la desescalada de la pandemia.

\section{DISCUSIÓN}

La tecnología puede proporcionarnos distintas opciones de comunicación a las consultas presenciales. Se deno- 
Tabla 1: Distribución de patologías más frecuentes según prioridad médica.

\begin{tabular}{lrc}
\hline Urgentes & Preferentes & Demorables \\
\hline $\begin{array}{l}\text { Casos oncológicos que han finalizado } \\
\text { neoadyuvancia }\end{array}$ & $\begin{array}{l}\text { Colelitiasis con antecedente de } \\
\text { colecistitis o pancreatitis aguda } \\
\text { Pacientes que requieren pruebas que } \\
\text { determinarán una cirugía preferente }\end{array}$ & Hernias y eventraciones muy sintomáticas \\
& $\begin{array}{c}\text { Colelitiasis no complicadas } \\
\text { Cirugía benigna de la mama } \\
\text { Cirugía proctológica benigna }\end{array}$ \\
\hline
\end{tabular}

mina telemedicina a aquélla que puede realizarse por vía telefónica, e-mail, videoconferencia, mensaje de texto, aplicaciones móviles, etcétera. La elección de ésta debe adaptarse al contexto en que nos encontramos y al tipo de población que tratamos. ${ }^{5}$

La telemedicina permite la comunicación con pacientes o entre personal médico para compartir guías de tratamiento, mecanismos de prevención, formación o realizar seguimiento de consulta. Su rol durante la pandemia COVID es fundamental para evitar riesgos de contagio innecesarios y liberar de presión al hospital. ${ }^{6}$

La consulta telefónica es una alternativa prometedora, se considera similar a las consultas presenciales en cuanto a resultados para el manejo de la promoción de la salud, el triaje y el seguimiento de enfermedades crónicas. Es una buena vía de comunicación y puede reducir la necesidad de consultas presenciales. El grado de satisfacción suele ser similar, aunque influirá la facilidad de acceso. ${ }^{7}$ Además, conlleva un ahorro de tiempo y económico para el paciente (costes de desplazamiento y pérdida de jornada laboral). Por este motivo, también nos podría permitir llegar a regiones o países con poblaciones más empobrecidas o difícil acceso al sistema sanitario. ${ }^{8}$

No todas las consultas pueden realizarse por esta vía, ya que perdemos la capacidad de la exploración física, lo cual nos deja a expensas de un único sentido, el oído. ${ }^{2}$ También existen limitaciones en la cantidad de información que se puede transmitir a través de video o audio. ${ }^{1}$ Asimismo, la barrera lingüística puede representar otro problema al lidiar con población inmigrante que no conoce el idioma local. Esto puede producir un impacto en la calidad y seguridad; por ello es importante realizar un triaje previo para seleccionar el tipo de consulta más adecuado para cada sujeto. ${ }^{5}$ El fácil acceso puede conllevar un aumento en la demanda, que no podemos dejar de tener en cuenta. ${ }^{8}$

Por teléfono también pueden realizarse cuestionarios que valoren el riesgo de infección COVID en el paciente que acudirá a consulta para, de esta forma, evitar riesgos añadidos. ${ }^{9}$
Estas iniciativas han mostrado que pueden reducir costes y tiempos de visita, y pueden aumentar la satisfacción de los pacientes. Debemos aprender a optimizarlas para maximizar la eficiencia y efectividad. ${ }^{1}$

Estudios realizados en sujetos con algunas enfermedades crónicas han reportado disminución de la mortalidad e ingresos hospitalarios en insuficiencia cardiaca y reducción de la hemoglobina glicosilada en diabetes tipo 2. Reduce recursos y mejora los resultados de los pacientes, con una satisfacción comparable. ${ }^{10}$

Bunn y colaboradores realizaron una revisión sistemática con varios niveles de evidencia. En una de las investigaciones se estimaba una reducción del 39\% del número de casos que requerían consultas presenciales después de una telefónica. ${ }^{11}$

Chapman y su equipo observaron en una revisión sistemática con varios niveles de evidencia que tanto pacientes como médicos la consideran una alternativa apropiada y que podría ayudar a reducir el número de consultas presenciales. ${ }^{12}$

McKinstry y su grupo de investigadores realizaron un ensayo clínico aleatorizado de 388 pacientes en Edimburgo (Reino Unido) de consulta presencial vs telefónica, analizando la utilización de recursos. Evidenciaron una reducción de 1.5 minutos en el tiempo de consulta (0.62.4) y un incremento de 0.2 (0-0.3) más de consultas de seguimiento en la telefónica. ${ }^{13}$

\section{CONCLUSIONES}

En la actualidad, es difícil establecer conclusiones estadísticas dada la poca evidencia existente en la literatura; sin embargo, en pacientes bien seleccionados, la telemedicina se muestra como una opción viable y ventajosa, que puede reducir tiempo, costes y aumentar el grado de satisfacción, además de permitir minimizar el riesgo de infección. A pesar de que su uso no está muy extendido, es una variante que se debe tener en cuenta de cara al futuro, sobre todo mientras dure la crisis COVID. 


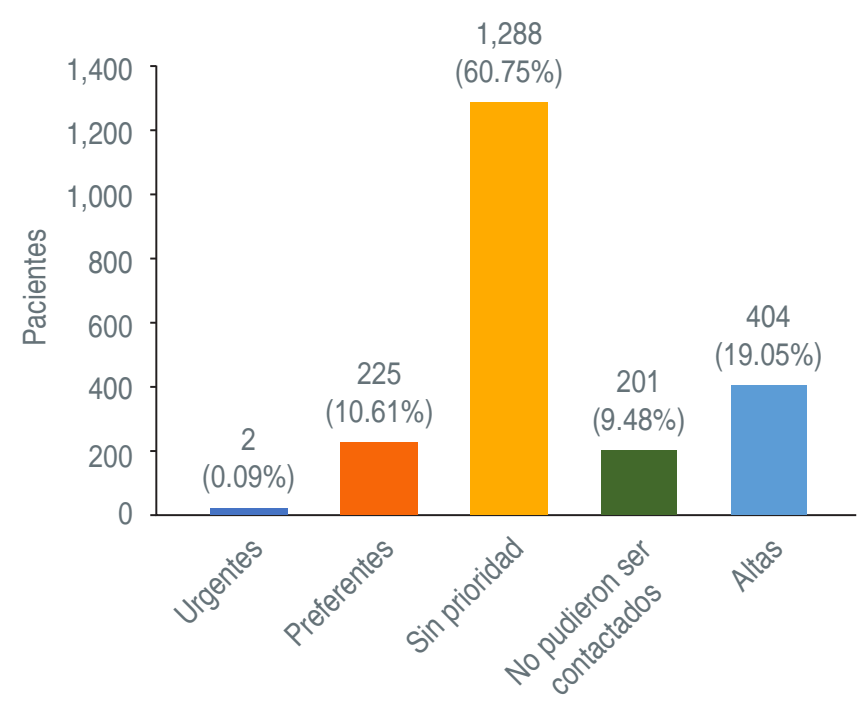

Figura 1: Distribución de los pacientes contactados.

En nuestro caso, la práctica de la consulta telefónica ha resultado beneficiosa para mantener el seguimiento de las consultas durante los meses de mayor intensidad de la crisis COVID, y para establecer un sistema de prioridad que nos permita reevaluar a cada persona según su grado de gravedad cuando se retomen las consultas presenciales. Las ventajas demostradas en ciertos casos nos plantean la posibilidad de mantenerla en un futuro en casos seleccionados.

\section{AGRADECIMIENTOS}

A todo el Servicio de Cirugía General del Hospital Universitario del Henares (Madrid), que ha participado de manera activa en el seguimiento telefónico.

\section{REFERENCIAS}

1. Prasad A, Brewster R, Newman JG, Rajasekaran K. Optimizing your telemedicine visit during the COVID-19 pandemic: Practice guidelines for patients with head and neck cancer. Head Neck. 2020; 42: 1317-1321.
2. Rajasekaran K. Access to telemedicine-are we doing all that we can during the COVID-19 pandemic? Otolaryngol Head Neck Surg. 2020; 163: 104-106.

3. COVIDSurg Collaborative. Global guidance for surgical care during the COVID-19 pandemic. Br J Surg. 2020: 10.1002/ bjs.11646.

4. Burki TK. Cancer guidelines during the COVID-19 pandemic. Lancet Oncol. 2020; 21: 629-630.

5. Atherton H, Brant H, Ziebland S, Bikker A, Campbell J, Gibson A et al. The potential of alternatives to face-to-face consultation in general practice, and the impact on different patient groups: a mixed-methods case study. Southampton (UK): NIHR Journals Library; 2018.

6. Song X, Liu X, Wang C. The role of telemedicine during the COVID-19 epidemic in China -Experience from Shandong province. Critical Care. 2020; 24: 178.

7. Downes MJ, Mervin MC, Byrnes JM, Scuffham PA. Telephone consultations for general practice: a systematic review. Syst Rev. 2017; 6: 128.

8. Griffiths F, Watkins JA, Huxley C, Harris B, Cave J, Pemba $S$ et al. Mobile consulting (mConsulting) and its potential for providing access to quality healthcare for populations living in low-resource settings of low- and middle-income countries. Digit Health. 2020; 6: 2055207620919594.

9. Tolone S, Gambardella C, Brusciano L, Del Genio G, Lucido FS, Docimo L. Telephonic triage before surgical ward admission and telemedicine during COVID-19 outbreak in Italy. Effective and easy procedures to reduce in-hospital positivity. Int J Surg. 2020; 78: 123-125.

10. Rush KL, Howlett L, Munro A, Burton L. Videoconference compared to telephone in healthcare delivery: a systematic review. Int J Med Inform. 2018; 118: 44-53.

11. Bunn F, Byrne G, Kendall S. The effects of telephone consultation and triage on healthcare use and patient satisfaction: a systematic review. Br J Gen Pract. 2005; 55: 956-961.

12. Chapman JL, Zechel A, Carter YH, Abbott S. Systematic review of recent innovations in service provision to improve access to primary care. Br J Gen Pract. 2004; 54: 374-381.

13. McKinstry B, Walker J, Campbell C, Heaney D, Wyke S. Telephone consultations to manage requests for sameday appointments: a randomised controlled trial in two practices. Br J Gen Pract. 2002; 52: 306-310. 